\title{
Distinct gene expression profiles associated with clinical outcomes in patients with ovarian clear cell carcinoma and high-grade serous ovarian carcinoma
}

\author{
Huimei Zhou', Qian Liu ${ }^{1+}$, Xiaohua Shi ${ }^{2+}$, Yue Liu ${ }^{1+}$, Dongyan Cao ${ }^{1+}$ and Jiaxin Yang ${ }^{1 *}$
}

\begin{abstract}
Background: Ovarian clear cell carcinoma (OCCC) is the second most common ovarian cancer after serous carcinoma in Southeast Asia. OCCC has a more unfavourable clinical outcome due to a poor response to platinumbased chemotherapy compared with serous carcinoma. The identification of biomarkers related to the prognosis of OCCC is critically important for an improved understanding of the biology that drives OCCC progression and leads to poor outcomes. To detect differences in gene expression profiles between OCCC and high-grade serous ovarian carcinoma (HGSOC), twelve patients with OCCC and twelve patients with HGSOC were recruited in whom the pathological diagnosis was confirmed on surgically resected specimens.

Results: Compared with HGSOC, OCCC has 609 differentially expression genes, and 199 are significantly different $(P<0.05)$. These genes are involved in the cell cycle, apoptosis, DNA damage repair, the PI3K pathway and so on. There were 164 differentially expressed genes in the PI3K pathway. There were 35 overexpressed genes in OCCC, while there were 12 overexpressed genes in HGSOC. Among these differentially expressed genes, we found that the MET gene and the CCNE1 gene were overexpressed in OCCC and associated with a worse prognosis.

Conclusions: In conclusion, there are many differentially expressed genes in OCCC and HGSOC, which indicates that the two kinds of tumours differ greatly in tumourigenesis and provides a theoretical basis for targeted therapy in the future. Further studies need to be performed to clarify the association of the differentially expressed genes with the unfavourable prognosis in OCCC.
\end{abstract}

Keywords: Ovarian clear cell carcinoma, Gene expression profiles, High-grade serous ovarian carcinoma, Outcome

\section{Background}

Different histological subtypes of epithelial ovarian cancer have different molecular characteristics and clinical prognosis [1]. Ovarian clear cell cancer (OCCC) accounts for 10\% of epithelial ovarian cancers and is known to be typically

\footnotetext{
* Correspondence: yangjiaxin@pumch.cn

${ }^{+}$Qian Liu, Xiaohua Shi, Dongyan Cao, and Yue Liu contributed equally to this work.

'Department of Gynaecologic Oncology, Peking Union Medical College Hospital, 1 Shuaifuyuan, Dongcheng-qu, Beijing, People's Republic of China Full list of author information is available at the end of the article
}

resistant to platinum-based chemotherapy and associated with a poorer prognosis than the more common serous subtype [2]. Although the carcinogenic mechanism and chemoresistance of OCCC are still unclear, several genetic changes have been extensively studied. Compared with HGSOC, OCCC is usually negative for p53 mutations, and the frequency of breast cancer 1 or 2 (BRCA1/2) mutations is low $(6.3 \%)[3,4]$. Higher frequencies of AT-rich active domain 1A (ARID1A) and phosphatidylinositol-4,5-diphosphate 3-kinase catalytic subunit alpha (PIK3CA) mutations

(c) The Author(s). 2020 Open Access This article is licensed under a Creative Commons Attribution 4.0 International License, which permits use, sharing, adaptation, distribution and reproduction in any medium or format, as long as you give appropriate credit to the original author(s) and the source, provide a link to the Creative Commons licence, and indicate if changes were made. The images or other third party material in this article are included in the article's Creative Commons licence, unless indicated otherwise in a credit line to the material. If material is not included in the article's Creative Commons licence and your intended use is not permitted by statutory regulation or exceeds the permitted use, you will need to obtain permission directly from the copyright holder. To view a copy of this licence, visit http://creativecommons.org/licenses/by/4.0/ The Creative Commons Public Domain Dedication waiver (http://creativecommons.org/publicdomain/zero/1.0/) applies to the data made available in this article, unless otherwise stated in a credit line to the data. 
(36\%) have also been observed in OCCC patients $[5,6]$. Therefore, further search for new tumour markers of chemoresistance and the development of new therapeutic targets are needed to help identify the mechanism of OCCC and for the effective clinical management of OCCC. Our study aimed to identify the molecular landscape of OCCC compared with HGSOC using the NanoString PanCancer 770 gene Pathway Panel to evaluate changes in RNA expression and their association with clinical outcomes.

\section{Patients and methods}

\section{Patient data and clinicopathological features (Table 1)}

Medical records of women with a diagnosis of OCCC and HGSOC patients who were treated between December 2013 and April 2017 at Peking Union Medical College Hospital were reviewed retrospectively. This study was approved by the ethics committee of PUMCH. Clinical and pathologic data were obtained from the medical records. A total of 24 patients with OCCC and HGSOC without preoperative chemotherapy whose tumours were surgically resected and pathologically confirmed were recruited for this study.

Details of surgery were collected from the operative records. Optimal cytoreduction was defined as $<1 \mathrm{~cm}$ of residual disease at the time of cytoreductive surgery. All of the patients were followed up every 3 months for the first 3 years, every 6 months for the following 2 years, and then once the following year. Oncologic outcomes were evaluated by a gynaecologic oncologist. Clinicopathological characteristics of these patients, such as age, the International Federation of Obstetrics and Gynecology (FIGO) stage, treatment regimens, recurrence, progression-free survival (PFS), and overall survival (OS), were reviewed.

\section{NanoString nCounter analysis}

Formalin-fixed, paraffin-embedded tumour samples were identified, and specimens were reviewed for a pathologic

Table 1 Clinical characteristics of twenty-four patients

\begin{tabular}{lll}
\hline Clinical characteristic & OCCC $(N=12)$ & HGSOC $(N=12)$ \\
\hline Age (years) & $47(32 \sim 62)$ & $57.6(39 \sim 75)$ \\
Stage & 1 & 1 \\
IC & 1 & 0 \\
IIb & 1 & 0 \\
IIla & 1 & 0 \\
IIIb & 7 & 9 \\
IIIC & 1 & 2 \\
IVb & 6 & 7 \\
Disease status at the last follow-up & 5 \\
Alive with disease & 6 & \\
Dead with disease & & \\
\hline
\end{tabular}

diagnosis and dissected if necessary to ensure that $\geq 90 \%$ of the sample represented the tumour. Samples were processed for analysis on the NanoString nCounter Flex system using the 770 gene PanCancer Pathways Plus panel (606 critical genes from 13 canonical cancer pathways, 124 cancer driver genes, and 40 reference genes) per the manufacturer's instructions (NanoString Technologies, Seattle, WA).

NanoString expression analysis identifies the differentially expressed genes in the selected patient specimens. The histological specimens of the selected cases required an effective area $>1.5 * 1.5 \mathrm{~cm}$ and $3 \sim 510 \mu \mathrm{m}$ wax rolls, and puncture specimens increased the number of wax rolls according to the tissue area. The experimental procedure included total RNA extraction, sample QC, overnight hybridization, hybrid product elution purification, sample plate preparation, sample plate scanning, and output.

\section{Statistical analysis}

NanoString data and pathway analyses are described above. Genetic polymorphisms and clinicopathological parameters in OCCC and HGSOC were assessed using Pearson's chi-square test or Fisher's exact test. The univariable survival analysis was performed by the generation of Kaplan-Meier curves, and differences between the groups were assessed using the log-rank statistic. SPSS v24.0 (SPSS, Inc., Chicago, IL, USA) was applied for all analyses. $p$ values $<0.05$ were considered significant.

\section{Results}

\section{Clinical characteristics}

Between December 2013 and April 2017, twelve patients with OCCC and twelve patients with HGSOC received treatment at PUMCH. All patients were treated with primary debulking surgery/comprehensive staging and platinum-based adjuvant chemotherapy postoperatively. The baseline clinical data of all patients are shown in Table 1 . The average follow-up time of 24 patients was 27.1 months (4.2 49.6 months), and the average recurrence interval was 6.2 months ( 1 19.7 months).

\section{NanoString expression analysis identifies differentially expressed genes between OCCC and HGSOC}

We performed NanoString expression analysis with the NanoString nCounter Flex system using the 770 gene PanCancer Pathways Plus panel (606 critical genes from 13 canonical cancer pathways, 124 cancer driver genes, and 40 reference genes) to compare OCCC and HGSOC tumour tissue in the patient cohort described in Table 1.

Compared with HGSOC, OCCC has 609 differentially expressed genes, and 199 were significantly different $(P<0.05)$. The pathways involved included the cell cycle, apoptosis, chromatin modification, DNA damage repair, 
driver genes, the Hedgehog, JAK-STAT, MAPK, Notch, PI3K, Ras, TGF-beta, and Wnt pathways and transcriptional misregulation (Fig. 1).

\section{The PI3K pathway shows more dominant alterations in OCCC compared with HGSOC}

Cancer-related pathways may serve as potential targets for therapeutic intervention. In this analysis, we identified 164 differentially expressed genes in the PI3K pathway. A notable observation was the significantly higher expression of 35 genes in the tumour tissue of OCCC compared with HGSOC and the significantly lower expression of 12 genes in the tumour tissue of OCCC compared with HGSOC (Fig. 2). Among these differentially expressed genes, PI3K pathway analysis revealed that the MET gene and the CCNE1 gene were associated with worse clinical outcomes. These two genes appeared to be more highly expressed in OCCC compared to HGSOC (Fig. 3 and Fig. 4).

\section{Discussion}

Treatment regimens for OCCC and HGSOC are the same at present; however, the clinical outcome between them is very different. Many studies have reported that these two kinds of ovarian cancer have different origins $[6,7]$; therefore, new attractive therapeutic targets will focus on differences in the genetic expression between the two cancers. Due to the poor prognosis and chemotherapy resistance of OCCC patients, a reliable genetic diagnosis and targeted therapy are beneficial to patients with rare types of ovarian cancer. Clinical observation and genomic testing are both necessary in the exploration. Mutations in PIK3CA (32-

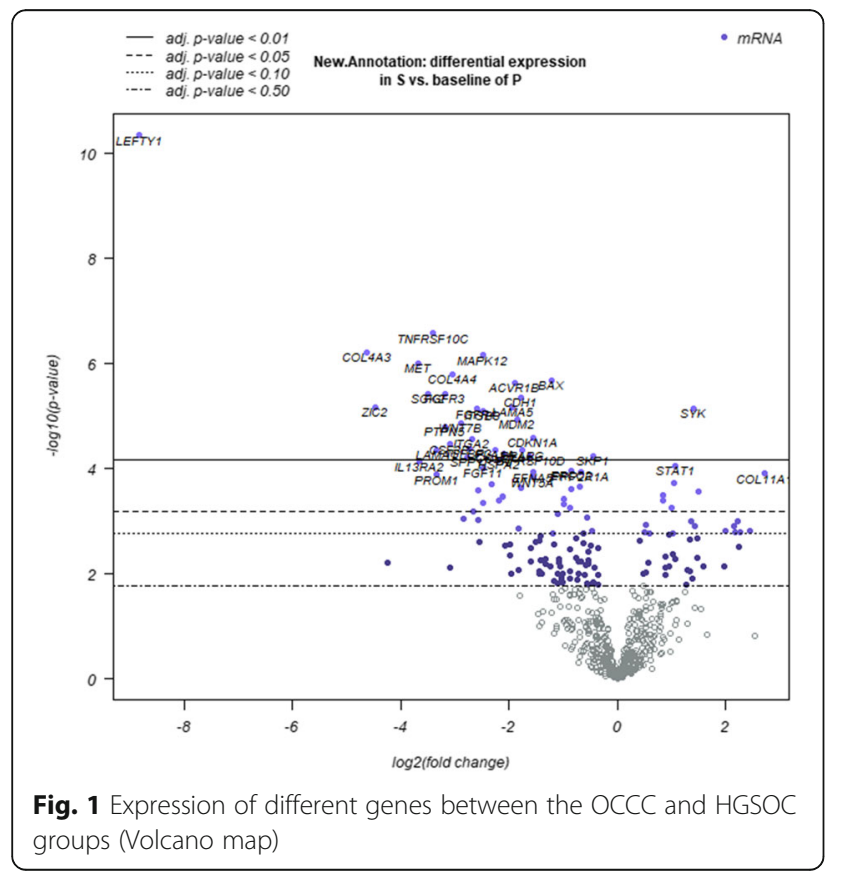

33\%), ARID1A (46\%), KRAS and BRAF are very common in the somatic mutation of OCCC $[8,9]$. The differentially expressed genes between these two subtypes of ovarian cancer are involved in multiple cancer pathways, including the cell cycle, apoptosis, chromatin modification, DNA damage repair, driver genes, the Hedgehog, JAK-STAT, MAPK, Notch, PI3K, Ras, TGF-beta, and Wnt pathways, and transcriptional misregulation. Furthermore, the PI3K pathway was one of the top pathways associated with these differentially expressed genes. Currently, targeted therapy for this pathway provides many new alternative options for the therapy of OCCC patients with a poor prognosis and treatment dilemma.

The MET proto-oncogene is located at chromosome $7 \mathrm{q} 31$ and encodes MET kinase, which is composed of three functional domains: the ligand-binding domain, the regulatory juxtamembrane domain, and the receptor tyrosine kinase domain [10]. In tumour cells, MET can be activated in a ligand-independent manner by mutating, amplifying or overexpressing the MET gene [11]. The MET signalling pathway is involved in processes of tumourigenesis, such as tumour proliferation, protection from apoptosis, angiogenesis, and motility [12]. Studies have confirmed that c-MET activation is associated with adverse clinical outcomes in lung, breast, stomach, kidney, and head and neck cancers $[13,14]$.

In EOC, MET is overexpressed in $7-27 \%$ of the population and is associated with progression and adverse outcomes of ovarian cancer [15]. Yamamoto et al. found that MET overexpression and gene amplification are ubiquitous in ovarian clear cell adenocarcinoma, with frequencies as high as $22-24 \%$ [16]. The MET protooncogene in OCCC participates in tumourigenesis through gene amplification. On the other hand, this change is extremely rare in nontransparent cell histological subtypes of ovarian cancer (i.e., serous, endometrioid, and mucinous adenocarcinoma). Recently, studies have reported that the c-MET amplification rate in OCCC is as high as $37.0 \%$ and is associated with poor survival [17]. MET overexpression is associated with a poor prognosis in patients with OCCC. Kim HJ et al. investigated the therapeutic effects of c-MET inhibitors on OCCC. The results showed that the inhibition of c-MET by c-MET inhibitors (SU11274 or crizotinib) significantly reduced OCCC cell proliferation and increased apoptosis. In vivo experiments confirmed that c-MET inhibitors can significantly reduce the tumour weight of the RMG1 cell xenograft model in vivo and the OCCC PDX model [10].

Compared with ovarian serous or endometrioid carcinoma, OCCCs have obvious chemotherapy resistance and poor prognoses. Previous studies have shown that stage I/II OCCCs have a better prognosis than HGSOC, while stage III/IV OCCCs have a worse prognosis than 


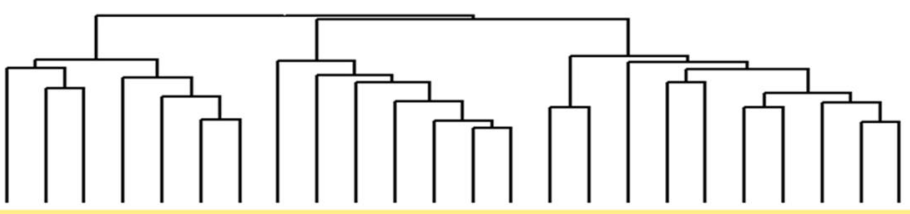
Positive. Control.QC.Flag
Binding.Density. QC.Flag

Imaging.QC.Flag
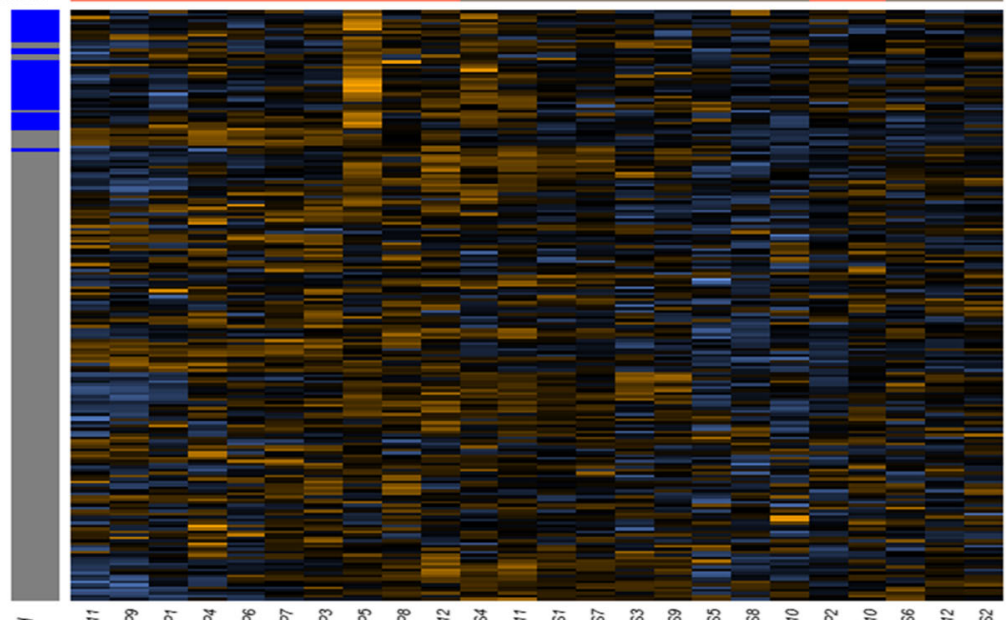

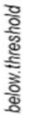

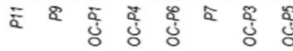

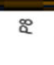

Fig. 2 Related differentially expressed genes involved in the PI3K pathway (heatmap)

HGSOCs [18]. The combination of Met and CD44 and CD47 may promote the progression of OCCCs [19]. OCCCs with Met amplification may have worse clinical outcomes. Therefore, alternative therapies for OCCCs, such as molecular targeted therapy, are urgently needed.

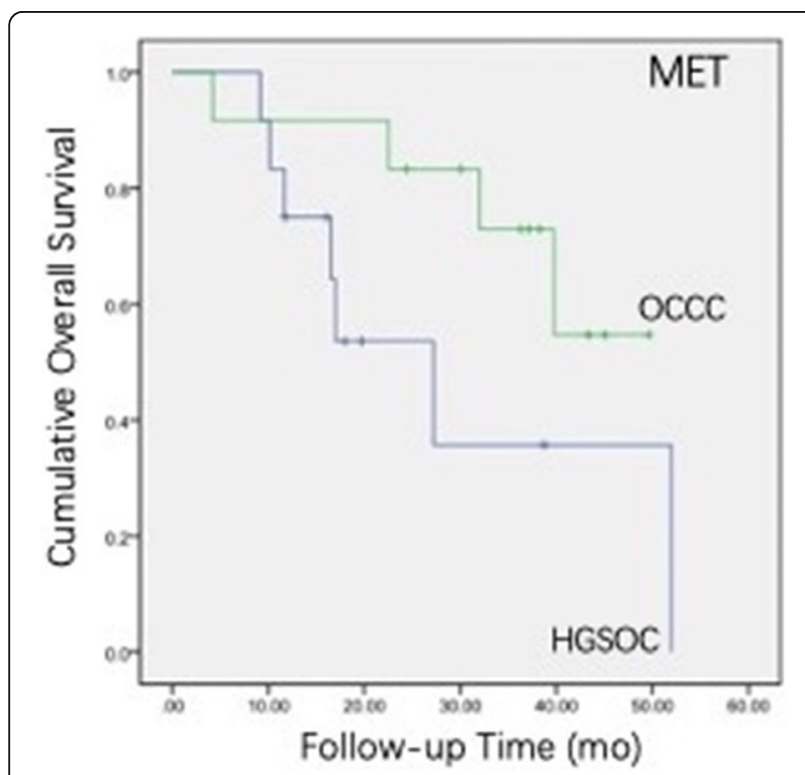

Fig. 3 Patients with a high MET level have a poor prognosis $(P=0.012)$
It is difficult to target the deletion of ARID1A, which often cooperates with the P53 gene. Therefore, Met is considered a good candidate for targeted therapy because of its high frequency of amplification and its high mutation specificity in OCCCs.

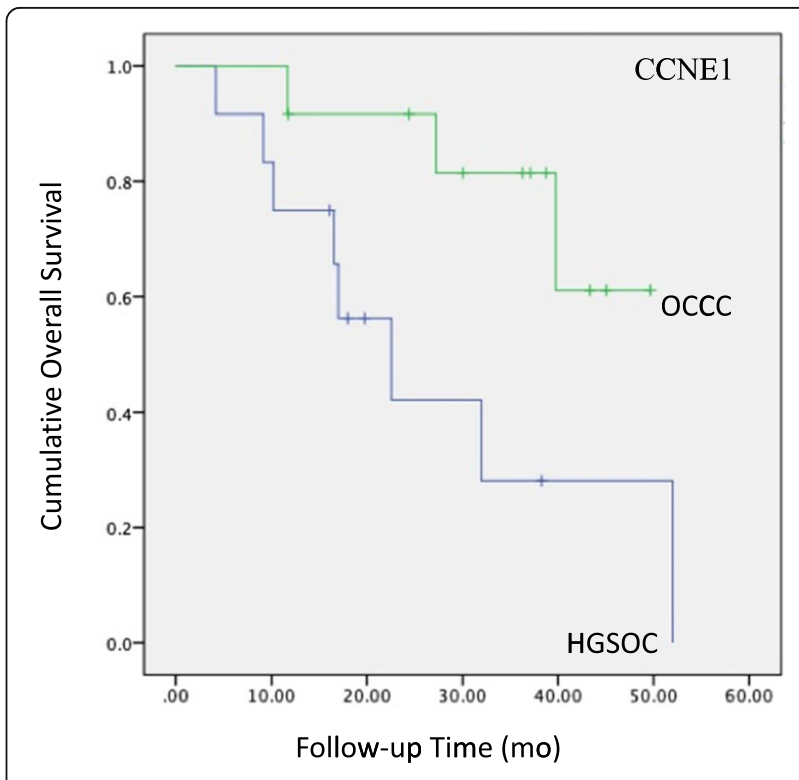

Fig. 4 Patients with a high CCNE1 level have a poor prognosis $(P=0.030)$ 
In this study, differentially expressed genes between OCCC and HGSOC and those associated with a poor prognosis were analysed by NanoString. The expression of MET was significantly different between OCCC and HGSOC; it was more highly expressed in OCCC compared to HGSOC. This is consistent with the results of previous studies. There was a significant correlation between high MET expression and good OS and between low MET expression and poor OS. The results should be confirmed further because of the relatively short follow-up time. It is still necessary to further verify the effects of the MET gene on the occurrence, development and drug resistance of OCCC at different levels and provide a more theoretical basis for the treatment of OCCC with the MET gene as a target.

The CCNE1 gene encodes the cell cycle E1 protein, which promotes the activity of cyclin-dependent kinase2 (Cdk2) and plays an important role in regulating the transition from $\mathrm{G} 1$ to $\mathrm{S}$ phase of the cell cycle [20]. One study reported that the CCNE1 gene also has direct action in triggering DNA replication and maintaining genomic stability [21]. Several studies have reported that CCNE1 gene amplification or protein upregulation is associated with higher tumour grades and a worse clinical outcome in a variety of cancers [22, 23]. CCNE1 overexpression is observed in ovarian high-grade but not lowgrade serous carcinomas. Exome sequencing identified $26 \%$ of OCCC patients with amplifications in the CCNE1 locus whose copy number gain was previously shown to be correlated with increased protein expression and associated with a worse outcome [24]. These results suggest the importance of CCNE1 in the progression of OCCC and support cyclin E1 as a possible therapeutic target in OCCC. In our study, we found that CCNE1 was more strongly overexpressed in OCCC patients than in HGSOC patients, which seems to be correlated with worse outcomes. These results suggest the importance of CCNE1 in the progression of OCCC and support cyclin E1 as a possible therapeutic target in OCCC.

As already stated, OCCC is the second most common ovarian cancer after serous carcinoma and represent $26 \%$ of ovarian cancer in Southeast Asia [3]. However, fewer molecular targets have been identified for OCCC compared with HGSOC. To the best of our knowledge, we present here the series with a distinct gene expression identification for these two histotypes of Chinese ovarian cancer for the first time. We found that MET and CCNE1 maybe play tumorigenesis roles in OCCC. These genes could be used as biomarkers and therapeutic targets for OCCC. Additional functional analysis for these genes is necessary to reveal new targets of OCCC.

We would like to acknowledge some of the limitations of the study. This study was retrospective and performed on a small but relevant patient population in that most of patients were in advanced stage at need population. Despite this limitation, these findings present an opportunity to rationally approach future clinical trials in the treatment of OCCC.

\section{Conclusions}

In general, the system identification of differentially expressed genes in OCCC and HGSOC will enlighten us on the differences in tumorigenesis and provides a theoretical basis for targeted therapy of OCCC in the future. Further studies need to be performed to clarify the association of the differentially expressed genes with the unfavourable prognosis in OCCC. The present and future results will be applied to the development of potential diagnostic and therapeutic options for OCCC.

\section{Abbreviations}

Cl: Confidence interval; FIGO: International Federation of Obstetrics and Gynecology; HR: Hazard ratio; IRB: Institutional Review Board; OCCC: Ovarian clear cell carcinoma; OS: Overall survival; PFS: Progression-free survival

\section{Acknowledgements}

We thank all patients for participating in the follow-up.

\section{Authors' contributions}

$\mathrm{HZ}$ designed the study, performed the data analyses and wrote the manuscript. JY designed the study, performed the data analyses, revised the manuscript. QL, XS, DC, and YL collected and checked the data. QL, XS, DC, and $Y L$ are equal contributors in the author group. The author(s) read and approved the final manuscript.

\section{Funding}

This study was funded by the Chinese Academy of Medical Sciences Initiative of Innovative Medicine (CAMS-2017-I2M-1-002).

\section{Availability of data and materials}

All data generated or analysed during this study are included in the published article.

Ethics approval and consent to participate

This study was approved by the Institutional Review Board of PUMCH.

Consent for publication

Not applicable.

Competing interests

The authors declare that they have no competing interests.

\section{Author details}

${ }^{1}$ Department of Gynaecologic Oncology, Peking Union Medical College Hospital, 1 Shuaifuyuan, Dongcheng-qu, Beijing, People's Republic of China. ${ }^{2}$ Department of Pathology, Peking Union Medical College Hospital, Beijing, People's Republic of China.

Received: 24 September 2019 Accepted: 31 March 2020

Published online: 15 April 2020

References

1. Tan DS, Kaye S. Ovarian clear cell adenocarcinoma: a continuing enigma. J Clin Pathol. 2007:60:355-60.

2. Okamoto A, Glasspool RM, Mabuchi S, et al. Gynecologic Cancer InterGroup (GCIG) consensus review for clear cell carcinoma of the ovary. Int J Gynecol Cancer. 2014;24:S20-5

3. Ji JX, Wang YK, Cochrane DR, et al. Clear cell carcinomas of the ovary and kidney: clarity through genomics. J Pathol. 2018;244:550-64. 
4. Alsop K, Fereday S, Meldrum C, DeFazio A, et al. BRCA mutation frequency and patterns of treatment response in BRCA mutation-positive women with ovarian cancer: a report from the Australian Ovarian Cancer Study Group. J Clin Oncol. 2012;30(21):2654-63 2012-07-20.

5. Wiegand KC, Shah SP, Al-Agha OM, et al. ARID1A mutations in endometriosis-associated ovarian carcinomas. New Engl J Med. 2010;363: 1532-43.

6. Kuo KT, Mao TL, Chen X, et al. DNA copy numbers profiles in affinitypurified ovarian clear cell carcinoma. Clin Cancer Res. 2010;16:1997-2008.

7. Matulonis UA, Sood AK, Fallowfield L, et al. Ovarian cancer. Nat Rev Dis Primers. 2016:2:16061.

8. Yamamoto S, Tsuda H, Takano M, Tamai S, Matsubara O. Loss ofARID1A protein expression occurs as an early event in ovarian clear-cell carcinoma development and frequently coexists with PIK3CAmutations. Mod Pathol. 2012;25:615-24.

9. Tan DS, Iravani M, McCluggage WG, et al. Genomic analysis reveals the molecular heterogeneity of ovarian clear cell carcinomas. Clin Cancer Res. 2011;17:1521-34

10. Kim HJ, Yoon A, Ryu JY, , et al. c-MET as a Potential Therapeutic Target in Ovarian Clear Cell Carcinoma. Sci Rep. 2016;6:38502.

11. Ma PC, Maulik G, Christensen J, et al. C-met: structure, functions and potential for therapeutic inhibition. Cancer Metastasis Rev. 2003;22:309-25.

12. Peruzzi B, Bottaro DP. Targeting the c-met signaling pathway in cancer. Clin Cancer Res. 2006;12:3657-60

13. Lengyel E, Prechtel D, Resau JH. C-met overexpression in node-positive breast cancer identifies patients with poor clinical outcome independent of Her2/neu. Int J Cancer. 2005;113:678-82.

14. Furlan A, Kherrouche Z, Montagne R, et al. Thirty years of research on met receptor to move a biomarker from bench to bedside. Cancer Res. 2014;74: 6737-44.

15. Sawada K, Radjabi AR, Shinomiya N, et al. C-met overexpression is a prognostic factor in ovarian cancer and an effective target for inhibition of peritoneal dissemination and invasion. Cancer Res. 2007;67:1670-9.

16. Yamashita Y, Akatsuka S, Shinjo K, et al. Met is the most frequently amplified gene in endometriosis-associated ovarian clear cell adenocarcinoma and correlates with worsened prognosis. PLoS One. 2013;8:e57724.

17. Bu R, Uddin S, Bavi P, et al. HGF/C-met pathway has a prominent role in mediating antiapoptotic signals through AKT in epithelial ovarian carcinoma. Lab Investig. 2011:91:124-37.

18. Kobel M, Kalloger SE, Huntsman DG, et al. Differences in tumor type in lowstage versus high-stage ovarian carcinomas. Int J Gynecol Pathol. 2010;29: 203-11.

19. Wang $\mathrm{H}$, Tan M, Zhang S, et al. Expression and significance of CD44, CD47 and c-met in ovarian clear cell carcinoma. Int J Mol Sci. 2015;16:3391-404.

20. Etemadmoghadam D, Au-Yeung G, Wall M, et al. Resistance to CDK2 inhibitors is associated with selection of polyploid cells in CCNE1-amplified ovarian cancer. Clin Cancer Res. 2013;19:5960-71.

21. Noske A, Henricksen LA, Lafleur B, et al. Characterization of the 19q12 amplification including CCNE1 and URI in different epithelial ovarian cancer subtypes [J]. Exp Mol Pathol. 2015:98:47-54.

22. Amininia S, Hashemi M, Ebrahimi M, et al. Association between CCNE polymorphisms and the risk of breast cancer in a sample of southeast Iranian population [J]. Med Oncol. 2014;31(10):189.

23. Ooi A, Oyama T, Nakamura R, et al. Gene amplification of CCNE1, CCND1, and CDK6 in gastric cancers detected by multiplex ligation dependent probe amplification and fluorescence in situ hybridization [J]. Hum Pathol. 2017:61(1):58-67.

24. Nakayama N, Nakayama K, Shamima Y, et al. Gene amplification CCNE1 is related to poor survival and potential therapeutic target in ovarian cancer [J]. Cancer. 2010;116:2621-34.

\section{Publisher's Note}

Springer Nature remains neutral with regard to jurisdictional claims in published maps and institutional affiliations.

\section{Ready to submit your research? Choose BMC and benefit from:}

- fast, convenient online submission

- thorough peer review by experienced researchers in your field

- rapid publication on acceptance

- support for research data, including large and complex data types

- gold Open Access which fosters wider collaboration and increased citations

- maximum visibility for your research: over $100 \mathrm{M}$ website views per year

At BMC, research is always in progress.

Learn more biomedcentral.com/submissions 